\title{
Correlative Characterization of Defect Clusters in GaAs Solar Cells by High- Resolution TEM and Spatially Resolved Optical Techniques
}

\author{
B.S. McKeon ${ }^{1}$, Q. Chen ${ }^{2}$, S. Zhang ${ }^{2}$, C.-K. $\mathrm{Hu}^{2,3}$, T.H. Gfroerer ${ }^{4}$, M.W. Wanlass ${ }^{5}$, Y. Zhang ${ }^{2}$, and David \\ J. Smith ${ }^{1}$ \\ 1. Department of Physics, Arizona State University, Tempe, Arizona, USA \\ 2. Department of Electrical and Computer Engineering, University of North Carolina at Charlotte, \\ Charlotte, NC, USA \\ 3. Wuhan University of Technology, Wuhan, Hubei, China \\ 4. Davidson College, Davidson, NC, USA \\ 5. National Renewable Energy Laboratory, Golden, CO, USA
}

The maximum efficiency of semiconducting devices, such as a solar cell, depends in large part on the presence and character of structural defects in the active material. It is generally known that defects degrade the device performance but the effect of an individual defect on a real device, how that effect depends on the operation of the device, and how that effect correlates to the atomic-scale structure of the defect are not understood. These questions are being addressed by investigating the impact of opticallyand electrically-active defect clusters in GaAs solar cells using high-resolution TEM to determine and analyze their atomic-scale defect structure. This study was done on a GaAs solar cell comprised of many independent sub-cells. The structure of the cell, from bottom to top, was as follows: a $p$-type GaAs substrate, 80-nm $p$-type GaAs buffer layer, 50-nm $p$-type GaInP back-surface confinement layer, 3- $\mu \mathrm{m}$ p-type GaAs absorber layer, 40-nm $n$-type GaAs emitter layer, 50-nm $n$-type GaInP window layer, and an 80-nm $n$-type GaAs contact layer. An electroluminescence (EL) imaging system was used to locate isolated defects that were photoelectrically active, which appeared as dark spots in the EL image. Their location was precisely measured relative to recognizable features nearby. A Nova 200 NanoLab dualbeam system, operating the ion beam (FIB) at $30 \mathrm{keV}$, was used to prepare TEM cross-section samples. After thinning to electron transparency, the FIB was further used at $5 \mathrm{keV}$ to remove remaining amorphized material. A Philips CM200-FEG high-resolution TEM, operated at $200 \mathrm{keV}$, was used for initial imaging, and atomic-resolution images were taken with an aberration-corrected JEOL ARM200F STEM at $200 \mathrm{keV}$.

Figures 1 (a) and (b) are TEM images of defect clusters identified by EL. The dark contrast indicates a cluster of defects extending downwards from the surface of the solar cell into the GaAs absorber layer. These defects do not reach all the way to the back-surface confinement layer. Figure 1 (c) is a bright field STEM image taken at higher magnification showing the area indicated by the red rectangle in (b). Figures 2 (a) and (b) are high-angle annular dark field (HAADF) STEM images taken at higher magnification of the areas indicated in figure 1 (c) by a red circle, and a yellow square, respectively. Figure 2 (a) shows $30^{\circ}$ and $90^{\circ}$ partial dislocations with an intrinsic stacking fault. A yellow Burgers circuit is included for each dislocation, and the general Burgers circuit drawn in red shows the resulting total Burgers vector. An extra plane of atomic columns associated with the $30^{\circ}$ partial dislocation is indicated with a blue line. There are three dislocations in figure 2 (b), and each corresponds to an extra plane of atomic columns marked in the figure with a blue line. The two Burgers circuits drawn in yellow surround $30^{\circ}$ partial dislocations connected by an intrinsic stacking fault. The general Burgers circuit in red shows that the net Burgers vector in this projection is a null vector. Thus, it is likely that a perfect screw dislocation split to form these two partial dislocations. With further analysis of the types and 
structures of individual defects in solar cell devices, and an analysis of these results in correlation with optical and electrical characterization of the same defects, better understanding of the impact of the individual defect on the device should be realized [1].

\section{References:}

[1] This work was supported by Army Research Office Grant \#W911NF-16-1-0263. The authors gratefully acknowledge the use of facilities within the John M. Cowley Center for High Resolution Electron Microscopy at Arizona State University.
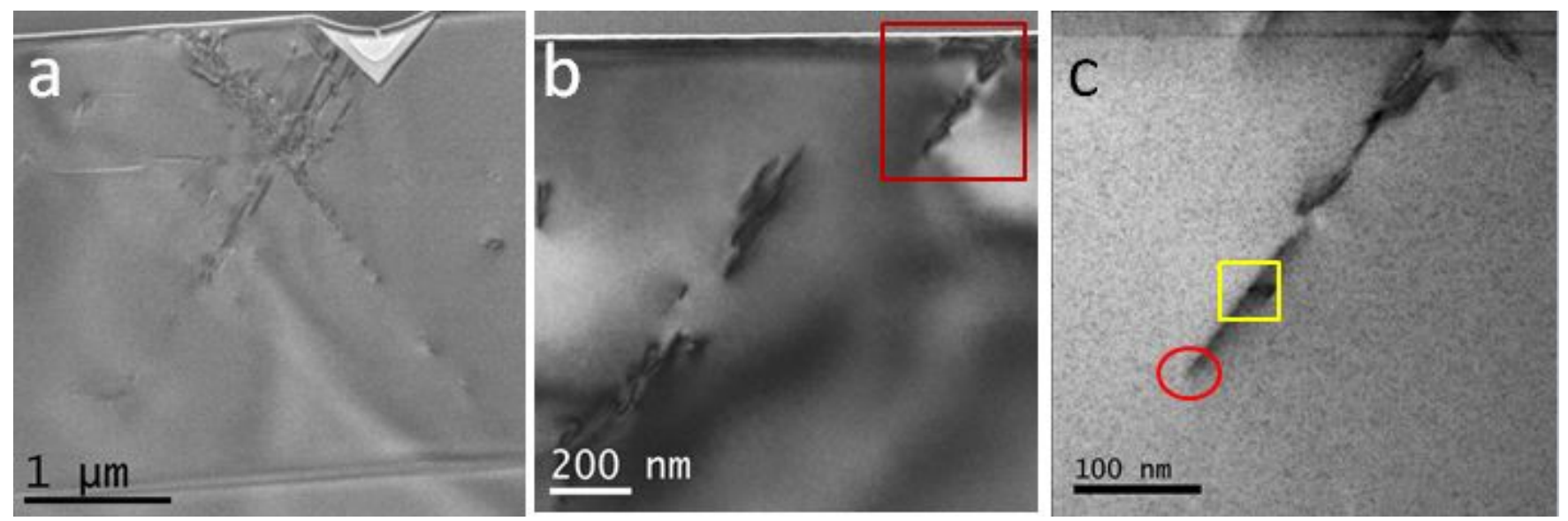

Figure 1. (a, b) TEM images of defect clusters identified by EL, (c) Higher magnification STEM image of the area highlighted in (b). The red circle and yellow square indicate the locations where figures 2 (a) and (b) where taken, respectively.
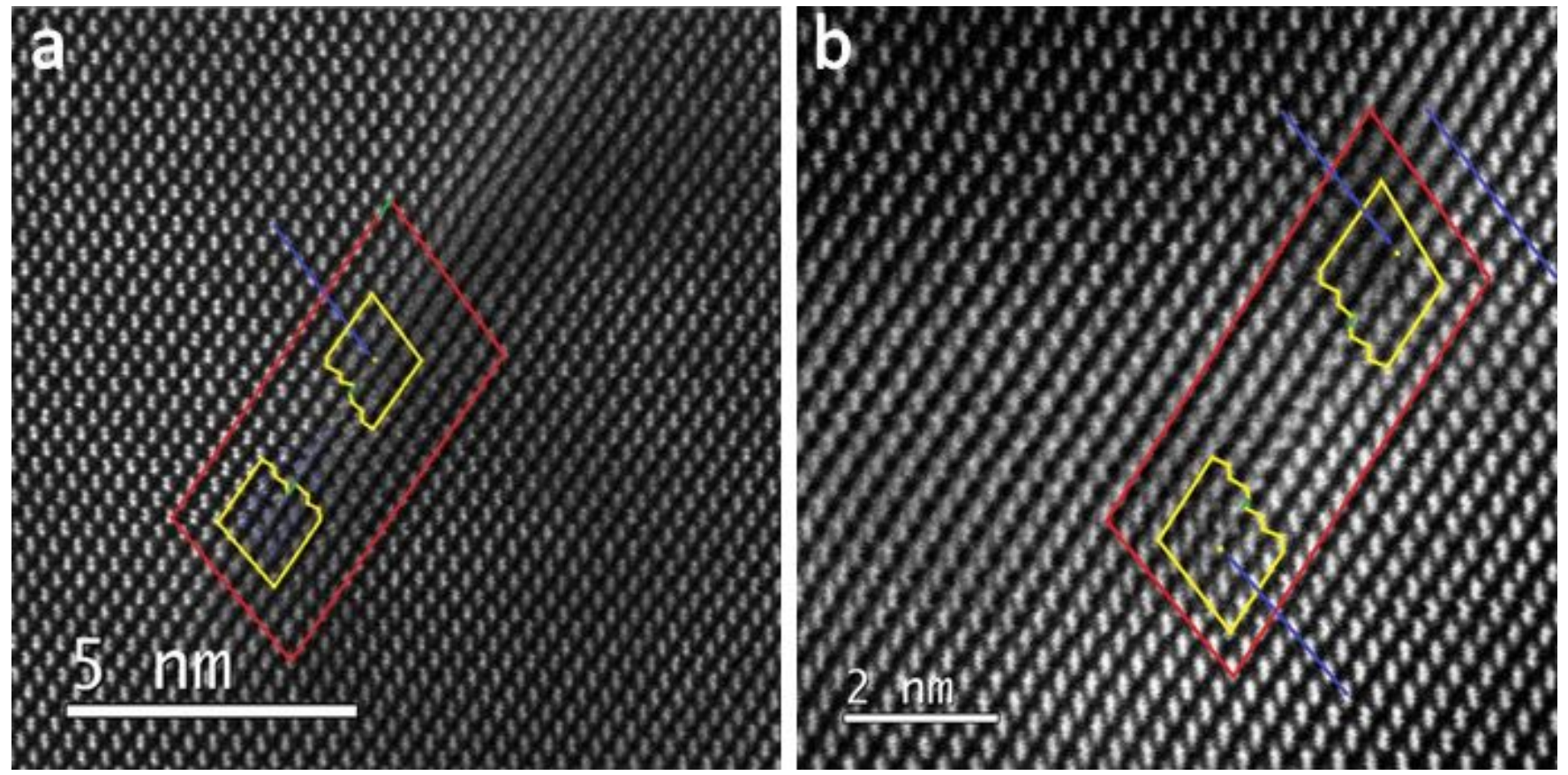

Figure 2. HAADF STEM images of individual defects. (a) $30^{\circ}$ and $90^{\circ}$ partial dislocations associated with an intrinsic stacking fault. (b) Two $30^{\circ}$ partial dislocations associated with an intrinsic stacking fault near another unidentified dislocation. 